University of Nebraska - Lincoln

DigitalCommons@University of Nebraska - Lincoln

Xia Hong Publications

Research Papers in Physics and Astronomy

2001

\title{
Epitaxial growth of $\mathrm{Pb}(\mathrm{Zr} 0.2 \mathrm{Ti0} .8) \mathrm{O} 3$ on $\mathrm{Si}$ and its nanoscale piezoelectric properties
}

A. Lin

X. Hong

V. Wood

A. A. Verevkin
C. H. Ahn
See next page for additional authors

Follow this and additional works at: https://digitalcommons.unl.edu/physicshong

Part of the Atomic, Molecular and Optical Physics Commons, and the Engineering Physics Commons

This Article is brought to you for free and open access by the Research Papers in Physics and Astronomy at DigitalCommons@University of Nebraska Lincoln. It has been accepted for inclusion in Xia Hong Publications by an authorized administrator of DigitalCommons@University of Nebraska Lincoln. 
Authors

A. Lin, X. Hong, V. Wood, A. A. Verevkin, C. H. Ahn, R. A. McKee, F. J. Walker, and E. D. Specht 


\title{
Epitaxial growth of $\mathrm{Pb}\left(\mathrm{Zr}_{0.2} \mathrm{Ti}_{0.8}\right) \mathrm{O}_{3}$ on $\mathrm{Si}$ and its nanoscale piezoelectric properties
}

\author{
A. Lin, X. Hong, V. Wood, A. A. Verevkin, and C. H. Ahn ${ }^{\text {a) }}$ \\ Department of Applied Physics, Yale University, New Haven, Connecticut 06520-8284 \\ R. A. McKee, F. J. Walker, and E. D. Specht \\ Oak Ridge National Laboratory, Oak Ridge, Tennessee 37831-6118
}

(Received 29 November 2000; accepted for publication 31 January 2001)

\begin{abstract}
We have demonstrated a route to epitaxial $\mathrm{Pb}\left(\mathrm{Zr}_{0.2} \mathrm{Ti}_{0.8}\right) \mathrm{O}_{3}$ on (001) Si that exhibits a uniform piezoelectric response down to nanoscale levels through the utilization of an insulating, single-crystalline $\mathrm{SrTiO}_{3}$ transition layer. These structures, which were grown by a combination of molecular-beam epitaxy and off-axis magnetron sputtering, have a surface roughness of $<5 \AA$, with piezoelectric microscopy measurements revealing a piezoelectric coefficient of $\sim 50 \mathrm{pm} / \mathrm{V}$ that is switchable down to sub-100-nm dimensions. (c) 2001 American Institute of Physics.
\end{abstract}

[DOI: $10.1063 / 1.1358848]$

Complex oxides exhibit a tremendous diversity of behavior, including magnetism, superconductivity, ferroelectricity, and piezoelectricity. The ability to integrate this versatile functionality in thin-film form with mainstream technology like Si processing opens broad opportunities for scientific and technological applications. For example, there has been much interest in interfacing polycrystalline and amorphous oxides and related materials with single-crystal Si for microelectronics applications, utilizing them as highdielectric-constant insulators to replace $\mathrm{SiO}_{2}$ as a gate oxide. $^{1}$

Here, we demonstrate another possibility, which is to couple atomically smooth, epitaxial ferroelectric oxides with single-crystal Si. In addition to their potential utility for microelectronics, these structures are also intrinsically piezoelectric, often with very large piezoelectric coefficients. This property makes them very attractive for microelectromechanical applications, such as sensing and actuation. ${ }^{2}$ They have also been investigated for high-density, nonvolatile, piezoelectric memory applications using scanning probes. ${ }^{3}$ The work presented here focuses on the nanoscale piezoelectric properties of such materials.

While there has been significant effort to grow these materials on $\mathrm{Si}$, to date, much of that work has focused on synthesis techniques like sol-gel processing and sputtering onto amorphous or polycrystalline surfaces, which produce amorphous or polycrystalline thin films. ${ }^{2,4}$ These results have demonstrated impressive bulk electromechanical coupling, but as progress is made towards smaller, nanoscale applications, for example, nanoelectromechanical systems (NEMS), where uniform control of the piezoelectric response over nanometer length scales is required, microstructural inhomogeneities in these polycrystalline films will eventually compromise performance. ${ }^{5}$

Ghonge et al. and Maruyama et al. have achieved crystalline growth of $\mathrm{Pb}_{0.9} \mathrm{La}_{0.1}\left(\mathrm{Zr}_{0.2} \mathrm{Ti}_{0.8}\right)_{0.975} \mathrm{O}_{3}$ and $\mathrm{Pb}\left(\mathrm{Zr}_{0.25} \mathrm{Ti}_{0.75}\right) \mathrm{O}_{3}$, respectively, on Si using a combination of

${ }^{\text {a)} E l e c t r o n i c ~ m a i l: ~ c h a r l e s . a h n @ y a l e . e d u ~}$ three buffer layers. ${ }^{6,7}$ However, in each case, one of the buffer layers is conducting and screens the $\mathrm{Si}$ from the ferroelectric polarization. Here, we demonstrate the epitaxial growth of $\mathrm{Pb}\left(\mathrm{Zr}_{0.2} \mathrm{Ti}_{0.8}\right) \mathrm{O}_{3}$ (PZT) on Si using an insulating $\mathrm{SrTiO}_{3}$ transition layer. With this composite dielectric structure on $\mathrm{Si}$, one can envision microelectronics applications where the polarization field of the ferroelectric modulates the electrical transport properties of the Si substrate.

These ferroelectric/Si heterostructures are nearly atomically smooth $[<5 \AA$ root-mean-square (rms) roughness $]$ and exhibit a highly uniform piezoelectric response, with isolated, sub-100-nm ferroelectric domains having been polarized and imaged. Figure 1 illustrates hysteresis data obtained for the $d_{33}$ piezoelectric coefficient of these materials measured using piezoelectric microscopy; the piezoelectric coefficient is on the order of $50 \mathrm{pm} / \mathrm{V}$, in agreement with measurements taken on epitaxial $\mathrm{Pb}\left(\mathrm{Zr}_{0.2} \mathrm{Ti}_{0.8}\right) \mathrm{O}_{3}$ grown on metallic, Nb-doped single-crystal $\mathrm{SrTiO}_{3}$ substrates. ${ }^{8}$ The details of the hysteretic shape of the data and charge modulation in the semiconductor will be discussed elsewhere; our primary consideration here is the piezoelectric response of these structures. ${ }^{9}$

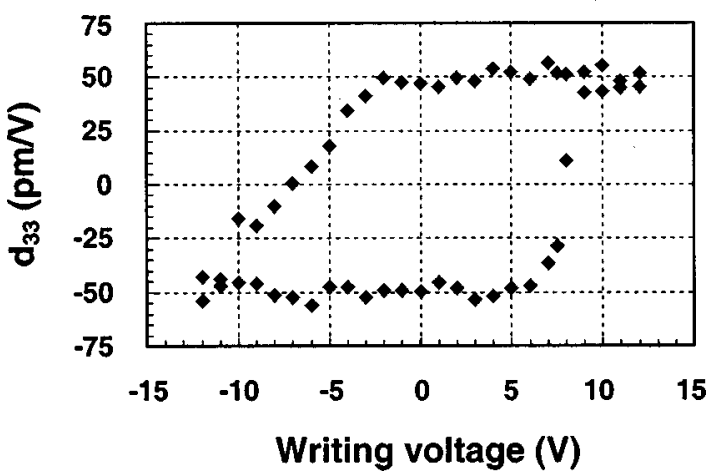

FIG. 1. Small-signal piezoelectric response of a $\mathrm{PZT} / \mathrm{SrTiO}_{3} / \mathrm{Si}$ heterostructure as a function of the writing voltage used to establish the ferroelectric polarization state. The zero of the piezoelectric response is defined as the mean of the positive and negative deflections measured at saturation. 
To carry out this work, we have employed a methodology for interfacing crystalline oxides with single-crystal $\mathrm{Si}$ (COS) developed by McKee and co-workers. ${ }^{10,11}$ This approach is centered around the layer-by-layer stabilization of the semiconductor-oxide interface, wherein the constituents of a crystalline oxide are grown in a sequenced transition from the covalently bonded semiconductor to the ionically bonded oxide. This technique can completely avoid amorphous $\mathrm{SiO}_{2}$ at the interface. It works well with the group IIA perovskites in the $\mathrm{CaTiO}_{3}$ class because the alkaline-earthoxide layer against silicon is itself stable, but it cannot be applied to $\mathrm{Pb}$-based perovskites since $\mathrm{SiO}_{2}$ is more stable than $\mathrm{PbO}$, preventing direct growth of single-crystalline PZT on Si. We have, therefore, used a variation of the COS approach by growing the stable $\mathrm{SrTiO}_{3}$ perovskite on silicon and then transitioning to PZT.

The $\mathrm{PZT} / \mathrm{SrTiO}_{3} / \mathrm{Si}$ structures we have investigated were grown by a combination of molecular-beam epitaxy (MBE) and off-axis magnetron sputtering. $\mathrm{SrTiO}_{3}$-terminated $\mathrm{Si}$ ( $p$ type) was first fabricated using the MBE methods described in Ref. 10. The samples were then transferred in air to an off-axis magnetron sputtering system, where epitaxial tetragonal PZT was deposited, as described in Refs. 12-16. Prior to deposition, the $\mathrm{SrTiO}_{3}$-terminated $\mathrm{Si}$ was held in the sputtering background gas at $500{ }^{\circ} \mathrm{C}$ for a few minutes to remove contaminants from the surface. No other surface treatment was necessary, indicating the robustness of the $\mathrm{SrTiO}_{3} / \mathrm{Si}$ surface. This procedure has also been used for the growth of $\mathrm{LaAlO}_{3}$ on $\mathrm{SrTiO}_{3} / \mathrm{Si}^{11}$

After growth, the structural properties of the $\mathrm{PZT} / \mathrm{SrTiO}_{3} / \mathrm{Si}$ heterostructures were characterized using x-ray diffraction. Figure 2(a) shows a $\theta-2 \theta$ scan, which reveals $c$-axis-oriented PZT on $\mathrm{SrTiO}_{3} / \mathrm{Si}$, with a PZT $c$-axis lattice parameter of $4.12 \AA$. While a small fraction of the film is $a$-axis oriented, no other impurity phases are detected. Figures 2(b) and 2(c) show off-axis $\phi$ scans of the PZT(111) and $\mathrm{SrTiO}_{3}(111)$ reflections. The fourfold symmetry of the scans and the alignment of the peaks in the two scans with respect to each other demonstrate epitaxial growth of PZT on $\mathrm{SrTiO}_{3} / \mathrm{Si}$. For this orientation of PZT, the ferroelectric polarization should lie parallel to the surface normal. Capacitance-voltage $(C-V)$ measurements taken on metaloxide-semiconductor capacitor structures fabricated from these samples confirm this direction of the polarization; a clockwise ferroelectric hysteresis in the $C-V$ data is observed, demonstrating the direct electrical coupling of the ferroelectric polarization to the charge state in the underlying p-type $\mathrm{Si}^{9}$

We have also used atomic-force microscopy (AFM) to examine the surface structure of these materials. Topographic imaging of a $2 \mu \mathrm{m} \times 2 \mu \mathrm{m}$ region reveals a surface with 4- $\AA$-rms roughness, the same as that of the $\mathrm{SrTiO}_{3}$ surface before the deposition of PZT. This scanning probe approach can also be used to examine the nanoscale piezoelectric properties of the $\mathrm{PZT} / \mathrm{SrTiO}_{3} / \mathrm{Si}$ structures. In this technique, which is described in detail elsewhere, a conducting AFM tip is scanned in contact with the surface of the sample. ${ }^{15,16}$ To define the local ferroelectric polarization state, and hence, the sign of the piezoelectric response, a voltage exceeding the coercive voltage is applied between
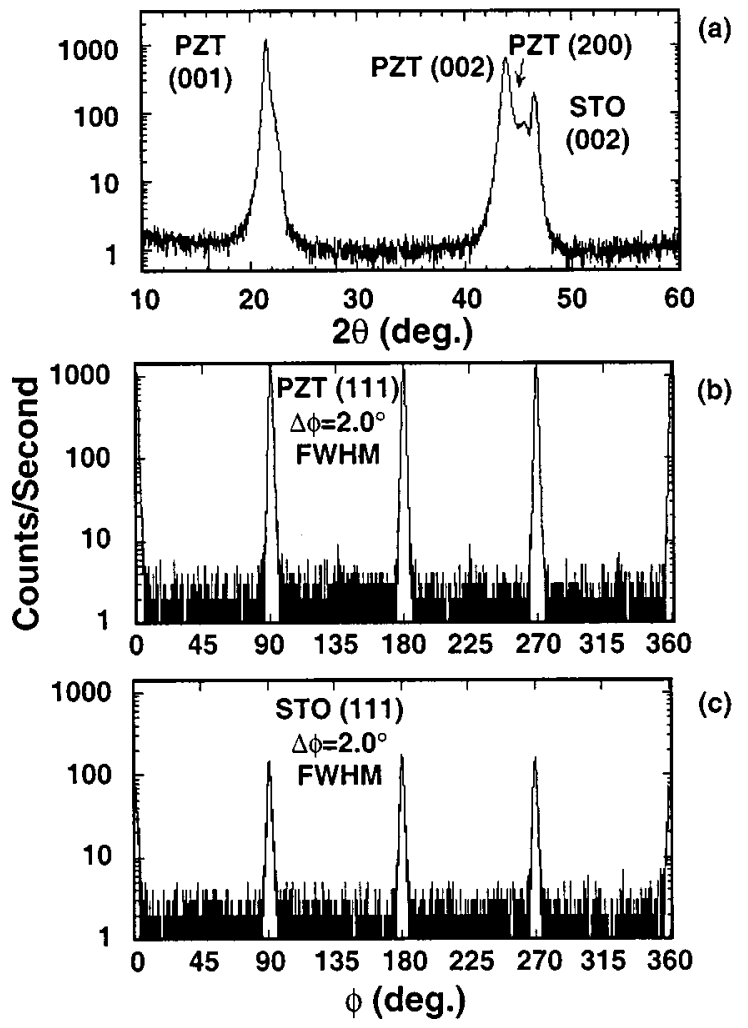

FIG. 2. X-ray characterization of a PZT/SrTiO $3 / \mathrm{Si}$ heterostructure. (a) $\theta-2 \theta$ diffraction scan of the thin-film heterostructure. (b) Off-axis $\phi$ scan showing the fourfold symmetry of the $\mathrm{SrTiO}_{3}$ film. (c) Off-axis $\phi$ scan showing the fourfold symmetry of the PZT film. The alignment of the PZT and $\mathrm{SrTiO}_{3}$ peaks demonstrates the epitaxial relationship between the two oxides.

the tip and the Si substrate during scanning. In order to image the resulting domain structure, the tip is scanned in contact with the sample while applying a small voltage (less than the coercive voltage) across the sample, inducing a local piezoelectric response. To increase the sensitivity of the measurement, standard ac lock-in detection is used for the deflection signal from the AFM.

With this technique, we have obtained the piezoelectric hysteresis loop shown in Fig. 1. To acquire these data, which were taken on a $500 \AA \mathrm{PZT} / 250 \AA \mathrm{SrTiO}_{3} / \mathrm{Si}$ heterostructure, a voltage was applied to a particular region of the sample to establish the local polarization state of the material, and the corresponding piezoelectric response was measured. By repeating this measurement for a series of different writing voltages, the ferroelectric hysteresis of the material is revealed.

The data in Fig. 1 demonstrate two distinct piezoelectric responses that correspond to the two polarization states of the material. The switching voltage we measure for the heterostructure is $\sim 7 \mathrm{~V}$, which is much larger than the expected coercive voltage of $0.5 \mathrm{~V}$ for the PZT layer itself (assuming a coercive field of $\sim 100 \mathrm{kV} / \mathrm{cm}$, the bulk value for $\left.\mathrm{Pb}\left(\mathrm{Zr}_{0.2} \mathrm{Ti}_{0.8}\right) \mathrm{O}_{3}\right)$. The discrepancy between the two values is due to the fact that most of the applied voltage drop occurs across the $\mathrm{SrTiO}_{3}$ layer in the heterostructure, as well as in the small (few $\AA$ ) vacuum gap that exists between the conducting AFM tip and the sample. ${ }^{14-16}$ One can estimate the value of the piezoelectric coefficient $d_{33}$ by considering the distribution of the fields in the structure. Assuming the dif- 


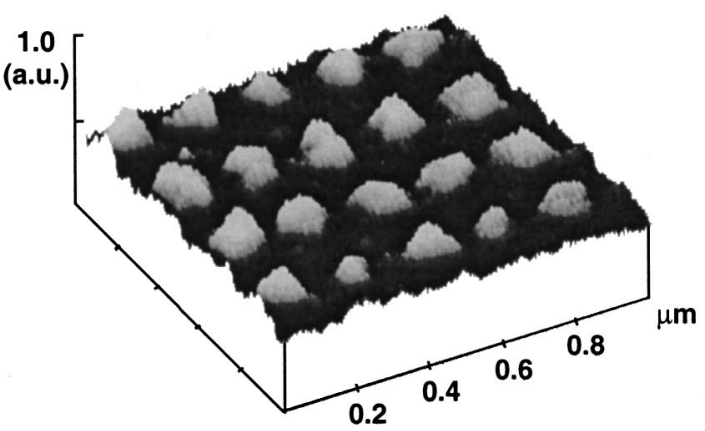

FIG. 3. Piezoelectric image of 20 small $(100 \mathrm{~nm})$ domains. The contrast in the image corresponds to the $0.5 \AA / \mathrm{V}$ piezoelectric response of the $\mathrm{PZT} / \mathrm{SrTiO}_{3} / \mathrm{Si}$ heterostructure.

ference in the observed coercive field and the expected coercive field $(100 \mathrm{kV} / \mathrm{cm})$ is due to the nonpiezoelectric layers, the value we find for the piezoelectric coefficient is $\sim 50$ $\mathrm{pm} / \mathrm{V}$, in agreement with quantitative measurements using scanning tunneling microscopy $(50 \mathrm{pm} / \mathrm{V})$ on epitaxial PZT thin films grown on Nb-doped single-crystal $\mathrm{SrTiO}_{3}$ substrates. ${ }^{8}$

We have also used this scanning probe microscopy approach to define and pattern the local piezoelectric properties over arbitrary, small regions of the film. Figure 3 shows a piezoelectric image of 20 polarized domains, which were written with $10 \mathrm{~V}$ inside an oppositely polarized background. The diameters of the 20 domains, as determined by the full width at half maximum of the piezoelectric signal, range from 80 to $120 \mathrm{~nm}$. The corresponding topographic image of this area is featureless, confirming that the domain contrast is indeed piezoelectric in origin. These domains are stable for at least seven days, the longest time we carried out measurements on these samples. We attribute the ability to write these small nanofeatures in a controlled fashion to the epitaxial, smooth surface of the films grown on the $\mathrm{SrTiO}_{3}$-terminated Si.

The robust piezoelectric response of these PZT films on $\mathrm{Si}$, combined with the high surface quality and highly controllable piezoelectric properties of these materials at the nanoscale level, offer several possibilities for applications ranging from NEMS, where uniform control of the piezoelectric response is required at the nanometer level, to highdensity, nonvolatile, piezoelectric memories using scanning probes. In addition, the direct electrical coupling of a ferro- electric to Si through an insulator enables one to envision extending this single-crystalline approach to microelectronics applications that utilize the polarization field of ferroelectrics to modulate the electronic properties of the $\mathrm{Si}$ semiconductor. ${ }^{9,17}$

The work at Yale University was supported by the Science Development Fund. The work at Oak Ridge National Laboratory was supported by the Division of Materials Sciences and Engineering, Office of Basic Energy Sciences, U.S. Department of Energy at Oak Ridge National Laboratory under Contract No. DE-AC05-00OR22725 with UTBattelle, LLC. The authors would like to thank T. P. Ma for useful discussions and V. Henrich for use of his laboratory space to carry out part of this work.

${ }^{1}$ See, for example, G. Lucovsky, J. Vac. Sci. Technol. A 18, 31 (2000).

${ }^{2}$ S. C. Minne, S. R. Manalis, and C. F. Quate, Appl. Phys. Lett. 67, 3918 (1995); T. Itoh and T. Suga, The Proceedigs of Transducers, '95 (Royal Swedish Academy of Engineering Science, IVA, Stockholm, Sweden, 1995), p. 632; T. Fujii, S. Watanabe, M. Suzuki, and T. Fujiu, J. Vac. Sci. Technol. B 13, 1119 (1995).

${ }^{3}$ T. Hidaka, T. Maruyama, M. Saitoh, N. Mikoshiba, M. Shimizu, T. Shiosaki, L. A. Wills, R. Hiskes, S. A. Dicarolis, and J. Amano, Appl. Phys. Lett. 68, 2358 (1996).

${ }^{4}$ G. Zavala, J. Fendler, and S. Trolier-McKinstry, J. Appl. Phys. 81, 7480 (1997).

${ }^{5}$ A. N. Cleland and M. L. Roukes, Nature (London) 392, 160 (1998).

${ }^{6}$ S. G. Ghonge, E. Goo, R. Ramesh, R. Haakenaasen, and D. K. Fork, Appl. Phys. Lett. 64, 3407 (1994); T. Maruyama, M. Saitoh, I. Sakai, T. Hidaka, Y. Yano, and T. Noguchi, ibid. 73, 3524 (1998).

${ }^{7}$ See also, T. Tybell, L. Antognazza, C. H. Ahn, L. Frauchiger, P. Pranyies, M. Decroux, M. G. Karkut, R. Passerini, P. Stadelmann, J. Ramm, E. Beck, Ø. Fischer, and J.-M. Triscone, Vide 283, 48 (1997).

${ }^{8}$ O. Kuffer, I. Maggio-Aprile, J.-M. Triscone, Ø. Fischer, and Ch. Renner, Appl. Phys. Lett. 77, 1701 (2000).

${ }^{9}$ R. A. McKee, F. J. Walker, and C. H. Ahn (unpublished).

${ }^{10}$ R. A. McKee, F. J. Walker, and M. F. Chisholm, Phys. Rev. Lett. 81, 3014 (1998).

${ }^{11}$ C. A. Billman, R. A. McKee, F. J. Walker, M. F. Chisholm, J. Lettieri, J. H. Haeni, and D. G. Schlom (unpublished).

${ }^{12}$ C. H. Ahn, J.-M. Triscone, N. Archibald, M. Decroux, R. H. Hammond, T. H. Geballe, Ø. Fischer, and M. R. Beasley, Science 269, 373 (1995).

${ }^{13}$ J.-M. Triscone, L. Frauchiger, M. Decroux, L. Miéville, Ø. Fischer, C. Beeli, P. Stadelmann, and G.-A. Racine, J. Appl. Phys. 79, 4298 (1996).

${ }^{14}$ C. H. Ahn, T. Tybell, L. Antognazza, K. Char, R. H. Hammond, M. R. Beasley, Ø. Fischer, and J.-M. Triscone, Science 276, 1100 (1997).

${ }^{15}$ See, for example, T. Tybell, C. H. Ahn, and J.-M. Triscone, Appl. Phys. Lett. 72, 1454 (1998), and references therein.

${ }^{16}$ T. Tybell, C. H. Ahn, and J.-M. Triscone, Appl. Phys. Lett. 75, 856 (1999).

${ }^{17}$ J. P. Han and T. P. Ma, Integr. Ferroelectr. 27, 9 (1999). 